\title{
Regelungsdefizite im Leichenschau- und Obduktionsrecht der Bundesrepublik Deutschland
}

Bei jedem Todesfall hat vor der Entscheidung über eine Obduktion zur Feststellung von Todesart und Todesursache eine auf landesrechtlicher Grundlage festgeschriebene »äußere Leichenschau « an der menschlichen Leiche durch einen approbierten Arzt stattzufinden. In den Bundesländern der ehemaligen DDR galt zunächst laut Einigungsvertrag $^{1}$ die alte »Anordnung über die ärztliche Leichenschau «. ${ }^{2}$ Bundeseinheitliche spezialgesetzliche Regelungen gem. $\S \S 87$ f. StPO, $§ 16$ Infektionsschutzgesetz (IfSchG), § 3 Feuerbestattungsgesetz (FeuerbestG) und $\S \S 103$ f. SGB VII (sozialversicherungsrechtliche Sektion) setzten diese alte Anordnung teilweise außer Kraft, ihre Vereinbarkeit mit dem Grundgesetz gilt im übrigen als zweifelhaft. ${ }^{3}$ Da der Bund für die Regelung der ärztlichen Leichenschau und den Erlaß eines Obduktionsgesetzes weder die ausschließliche noch die konkurrierende Gesetzgebungsbefugnis und auch nicht die Rahmengesetzgebungskompetenz besitzt, haben dafür die Länder die alleinige Gesetzgebungskompetenz, Art. 70 Abs. 1 GG. Mittlerweile sind in den letzten Jahren in einer Reihe von Bundesländern neue Bestattungsgesetze in Kraft getreten. ${ }^{4}$ Sowohl hinsichtlich der äußeren Leichenschau als auch bezüglich der »inneren Leichenschau « (Sektion, Obduktion, Autopsie) ist die Rechtslage jedoch uneinheitlich geblieben. ${ }^{5}$ Eine Definition des Begriffs »Leiche« findet sich u.a. in $\S 1$ des »Gesetzes über das Leichenwesen« der Freien Hansestadt Bremen. Danach ist jeder Körper einer verstorbenen Person eine Leiche, wenn der körperliche Zusammenhang noch nicht durch Verwesungsprozeß völlig aufgehoben ist. ${ }^{6}$ Davon abweichend gelten nach $\S 3$

1 Eine ausdrückliche Fortgeltung der Leichenschau-VO der ehemaligen DDR wurde in Art.9 Abs.2 EV i.V.m. der Anlage II nicht bestimmt, insofern richtet sich die Fortgeltung nach Art.9 Abs.1 EV.

2 DDR-GBl. 1979, S.4.

3 Einige halten die Leichenschau-VO der ehemaligen DDR für vereinbar mit dem Grundgesetz, etwa Hirsch/Schmidt-Didczuhn, Sektion und Transplantation, S. 43 ff. und Laufs, Die Entwicklung des Arztrechts 1991/92, NJW 1992, 1537 f.; skeptisch Steffen, Zur Strafbarkeit der klinischen Sektion gemäß § 168 StGB, S.39.

4 Vgl. z.B. Gesetz Nr. 1535 über das Friedhofs-, Bestattungs- und Leichenwesen, Amtsbl. d. Saarlandes v. 11. Dez. 2003, S. 2920 ff.; Thüringer Bestattungsgesetz v. 19. Mai 2004, GVBl. S. 505 f.; Gesetz über das Friedhofs- und Bestattungswesen (BestG NRW) v. 17. Juni 2003, GV. NRW Nr. 29 v. 30. Juni 2003, S. 313 ff.; Gesetz zur Regelung des Sektionswesens und therapeutischer Gewebeentnahmen (Sektionsgesetz) des Landes Berlin v. 18. Juni 1996, GVBl. Nr. 32, S. 237 ff., geänd. durch Gesetz zur Änderung des Sektionsgesetzes v. 7. März 1997, GVB1. Nr. 11, S. 54.

5 Siehe auch: Dettmeyer, Medizin \& Recht für Ärzte, 2001, S. 257-279.

6 »Gesetz über das Leichenwesen« der Freien Hansestadt Bremen vom 27. Oktober 1992, GB1. Nr.52, S.627, § 1 lautet vollständig: »(1) Menschliche Leiche im Sinne dieses Gesetzes ist der Körper einer verstorbenen Person, bei dem der körperliche Zusammenhang noch nicht durch Verwesungsprozeß völlig aufgehoben ist. Als menschliche Leiche gilt auch ein Körperteil, ohne den ein Weiterleben nicht möglich ist. Als menschliche Leiche gilt weiter 
Abs.1 S.2 des thüringischen Bestattungsgesetzes auch ein Kopf oder ein Rumpf (offenbar selbst in skelettierter Form) als Leiche. ${ }^{7}$

Abgesehen von den genannten bundesgesetzlich verankerten sog. Legalsektionen ist die Rechtsgrundlage für die Durchführung von Obduktionen teilweise umstritten und es finden sich landesgesetzlich unterschiedliche Regelungen. Nicht zuletzt diese Rechtsunsicherheit hat zu einer Obduktionsquote geführt, die derzeit allenfalls zwischen $3 \%$ und $4 \%$ aller Todesfälle liegen dürfte. ${ }^{8}$ Experten kritisieren seit Jahren, dass sich mit einer solchen Obduktionsquote auf Dauer weder eine Qualitätskontrolle der medizinischen Therapie, eine qualitativ hochwertige Aus- und Fortbildung der Mediziner auf pathologisch-anatomischem Gebiet, eine Kontrolle der Angabe zur Todesursache auf den Todesbescheinigungen nach der äußeren Leichenschau noch eine zuverlässige Beantwortung wissenschaftlich-epidemiologischer Fragestellungen gewährleisten läßt. ${ }^{9}$ Die gesetzliche Einführung einer sog. Verwaltungssektion zur Klärung der Todesursache bei medizinisch unklaren Todesfällen könnte Abhilfe schaffen. Mit einer derartigen Regelung - wie es sie in Österreich gibt und in der ehemaligen DDR gab - werden Sektionsfrequenzen von mehr als 50\% aller Todesfälle erreicht. ${ }^{10}$ Der Begriff der $»$ Verwaltungssektion $«$ ist nicht definiert. Obduktionen auf Veranlassung oder ausdrückliche Anordnung einer Behörde werden unter diesen Begriff gefaßt. Die Verwaltungssektion soll nach Ansicht ihrer Befürworter jene medizinisch unklaren Todesfälle erfassen, bei denen ein natürlicher Tod - d.h. ein Tod aus innerer Ursache ohne jedes Fremdverschulden - vorliegt, die Todesursache jedoch durch eine bloße äußere Leichenschau auch unter Heranziehung der Krankengeschichte nicht geklärt werden kann. Dabei kann eine Verwaltungssektion insbesondere auch jene Todesfälle außerhalb von Krankenhäusern und Kliniken einbeziehen, die nicht über eine klinische Sektion erfasst werden.

Anders als bei der Problematik der Explantation von Organen nach Eintritt des Hirntodes aus einem in seinen übrigen biologischen Funktionen bis zur Organentnahme noch (teilweise) intakten menschlichen Körper, erfolgt die Obduktion unstreitig an den »Überresten des Verstorbenen«, an seiner »leiblichen Hülle«, die Problematik des Zeitpunktes des Todeseintritts stellt sich ebensowenig wie die Frage,

der Körper eines Neugeborenen, bei dem nach vollständigem Verlassen des Mutterleibes, unabhängig vom Durchtrennen der Nabelschnur oder von der Aussstoßung der Plazenta 1. entweder das Herz geschlagen oder die Nabelschnur pulsiert hat (Lebendgeborenes) und das danach verstorben ist oder

2. keines der unter Nummer 1 genannten Lebenszeichen vorhanden war, das Geburtsgewicht jedoch mindestens $500 \mathrm{~g}$ betrug (Totgeborenes).

(2) Keine menschliche Leiche ist eine Leibesfrucht mit einem Gewicht unter $500 \mathrm{~g}$, bei der nach vollständigem Verlassen des Mutterleibes keine der in Absatz 1 Nr.1 genannten Lebenszeichen vorhanden war (Fehlgeborenes).«

7 ThürBestG v. 19. Mai 2004, GVBl. S. 505 ff.

8 Brinkmann/Du Chesne/Vennemann, Aktuelle Daten zur Obduktionsfrequenz in Deutschland, Dtsch Med Wschr 127 (2002), S. 791-795.

9 Siehe Madea/Dettmeyer, Obduktionen, in: Madea (Hrsg.) Die ärztliche Leichenschau, S. $217 \mathrm{ff}$.

10 Brinkmann/Kleiber/Janssen, Pathologe 2 (1981), S. $201 \mathrm{ff}$. 
ob der »Hirntote « als »Leichnam « zu qualifizieren ist. ${ }^{11}$ Gesetzliche Regelungen von Leichenschau und insbesondere der Obduktion werfen Fragen nach der Rechtsnatur des Leichnams ${ }^{12}$ bzw. verfassungsrechtliche Fragen auf: Wird der Verstorbene beziehungsweise der Leichnam selbst noch vom Schutzbereich der Grundrechte erfasst, ist er möglicherweise selbst Grundrechtsträger? Nehmen die Hinterbliebenen des Verstorbenen dessen grundrechtlich geschützte Interessen »treuhänderisch « wahr oder sind sie durch eine Obduktion gar in ihren eigenen Grundrechten betroffen? Ist der Verstorbene beziehungsweise der Leichnam kein Grundrechtsträger, so wäre zu fragen, wie sich dann der allgemein anerkannte Respekt vor der Würde des Verstorbenen bzw. vor dem Leichnam als postmortal fortwirkend verfassungsrechtlich begründen läßt. Soweit dem Leichnam oder den totensorgeberechtigten Hinterbliebenen des Verstorbenen grundrechtlich begründete Rechte oder auch Schutzansprüche gegen den Staat zugestanden werden, stellt sich das Problem, inwieweit der Gesetzgeber die gesetzliche Ermächtigungsgrundlage schaffen darf für die behördliche Anordnung einer Obduktion zur Klärung der medizinisch unklaren Todesursache. ${ }^{13}$ Hinsichtlich der gesetzlich festzulegenden Voraussetzungen einer solchen (Verwaltungs-) oder klinischen Sektion bieten sich - wie im Transplantationsrecht - alternative Lösungsmodelle an, von der »Einwilligungslösung « über die »Informationslösung « bis zur »Widerspruchslösung $« .{ }^{14}$

\section{Entstehung und Entwicklung des Leichenschau- und Obduktionswesens}

Die Anfänge der ärztlichen Leichenschau für forensische Zwecke reichen im europäischen Kulturkreis zurück bis in das Mittelalter. Der Sachsenspiegel erwähnt die forensische Leichenschau ${ }^{15}$, die ersten nicht forensischen Leichenöffnungen sollen um 1240 in Salerno durchgeführt worden sein. ${ }^{16}$ Als Teil des strafprozessualen Ermittlungsverfahrens ordnete die Bambergische Peinliche Halsgerichtsordnung in der Fassung von 1508 in Artikel 173 die Konsultation von Ärzten in »zweifelhaften Todesfällen« an. Artikel 147 der Constitutio Criminalis Carolina von 1532 verlangte

11 Dazu Höfling, JZ 1995, S. 26 (31); Höfling in Sachs, Grundgesetz, Art.1 Rdnr. 55.

12 Dettmeyer, Rechtsnatur des Leichnams, in: Brinkmann/Madea (Hrsg.) Handbuch gerichtliche Medizin, Bd. 1, S. $18 \mathrm{ff}$.

13 Ausführlicher: Dettmeyer, Die verfassungsrechtlichen Grenzen für die gesetzliche Einführung einer Verwaltungsssektion bei medizinisch unklaren Todesfällen, Bochumer Schriften zum Sozialrecht (BOSS), Bd. 3, Frankfurt a.M. 1999.

14 Diese Lösungsmodelle werden im Zusammenhang mit einer Regelung der Sektionsproblematik u.a. diskutiert von Hirsch/Schmidt-Didczuhn, S. 66; Steffen, S. 150 ff.; Empfehlung der DGMR, MedR. 1991, S. 76; Mohr, Pathologe 2 (1981), S. 139; Dettmeyer, Fn. 13, S. $201 \mathrm{ff}$.

15 Homeyer, Des Sachsenspiegels Erster Theil oder das Sächsische Landrecht, nach der Berliner Handschrift v.J. 1369, Drittes Buch, Neunzigster Artikel, § 2.

16 Hintsche, Anatomische Forschungen im mittelalterlichen Abendland, Ciba-Zschr. 8 (1944), S. 3426 ff.; siehe auch: Artelt, Die ältesten Nachrichten über die Sektion menschlicher Leichen im mittelalterlichen Abendland, Abh.z.Gesch.d.Med.u.d. Naturwissenschaften. Heft 34, Berlin 1940. 
eine Leichenschau »so eyner geschlagen wirdt und stirbt und man zweiffelt, ob er an den Wunden gestorben sei.« Zugleich ordnete Artikel 149 die »Besichtigung eynes entleibten vor der begrebnu $\beta$ « an. ${ }^{17} \mathrm{Im}$ Jahre 1754 wurde in Wien eine allgemeine Leichenschau durch Wundärzte der damaligen Zeit angeordnet, 1806 gab es eine vergleichbare Regelung in Stettin. In Preußen wurde kurz vor den Befreiungskriegen ein Totenschein eingeführt für die im Lazarett verstorbenen Soldaten, 1824 dann in Berlin ein genereller Sterbezettel. ${ }^{18} 1835$ ordnete der Berliner Polizeipräsident die Durchführung einer obligatorischen ärztlichen Leichenschau an. In Preußen betrafen Erlasse vom 9.6.1889 und 10.12.1889 die Übergabe von Leichen Strafgefangener an anatomische Institute. Diese Übergabe sollte dann erfolgen können, wenn die Angehörigen nicht widersprochen hatten. ${ }^{19}$ Ein sächsisches Gesetz vom 5.10 .1912 betraf die Ablieferung von Leichen zu wissenschaftlichen Zwecken und die Öffnung von Leichen. ${ }^{20}$ Im Jahre 1932 wies ein Erlaß des Preußischen Ministers des Innern zur Regelung von Obduktionen die Krankenhausleitungen darauf hin, daß »es sich empfiehlt, sich des Einverständnisses der Angehörigen des Toten zu vergewissern $\ll .{ }^{21} 1937$ forderte die Deutsche Gesellschaft für Gerichtliche und Soziale Medizin die reichsgesetzliche Einführung von Verwaltungssektionen in allen Fällen, in denen der zuständige Leichenschauer keine »einwandfreie Todesursache « feststellen konnte. ${ }^{22}$ Diese Forderung wurde seinerzeit vom Gesetzgeber nicht aufgegriffen. Nach dem Zweiten Weltkrieg wurde auf Landesebene den niedergelassenen Ärzten die Leichenschau übertragen. Im Jahre 1976 erwog die Mehrheit einer Bund-Länder-Arbeitsgruppe eine gesetzliche Pflichtsektion. ${ }^{23}$ Seit 1977 gibt es durch entsprechende - wenn auch recht uneinheitliche - Regelungen auf Landesebene im gesamten Bundesgebiet eine obligatorische ärztliche Leichenschau. ${ }^{24}$ Die vom Leichenschauarzt im Anschluß an die Leichenschau festzulegende Todesart wird im Grundsatz unterteilt in drei Gruppen: »natürlich«, »nicht-natürlich« und »ungeklärt« (nicht in allen Bundesländern!). In der ehemaligen DDR regelte die »Anordnung über die ärztliche Leichenschau« vom 4.12.1978 Fragen des Leichenschaurechts und der Leichenöffnung. ${ }^{25}$ Darin waren einheitliche Totenscheinformulare vorgesehen und die Möglichkeit einer angeordneten

17 Vgl. Radbruch, Die Peinliche Gerichtsordnung Kaiser Karls V von 1532 (Carolina); Stürzbecher, Über die Entwicklung der Leichenschau in Berlin, Beitr. ger. Med. 1970, S. 256 f.

18 Schneider, Die Leichenschau, S. 4.

19 Preuß. Min.B1. 1889, S.133 und 222.

20 GuVOBl. Kgr. Sachsen 1912, S. 465; vgl. Weise, Das sächsische Anatomiegesetz, DJZ 1912, S. $1292 \mathrm{f}$.

21 Erlaß vom 22.12.1932, zit. nach Franzki, Medizinrechtliche Probleme der Obduktion und Sektion, MedR. 1991, S. 223.

22 Merkel, Über die Notwendigkeit der Einführung von Verwaltungssektionen und deren Durchführbarkeit, Dt. Zschr. ges. gerichtl. Med. 28 (1937), S. 1-21.

23 Siehe Bericht der Bund-Länder-Arbeitsgruppe, S. 24/25.

24 Lediglich auf einigen schleswig-holsteinischen Inseln ist die Leichenschau durch vertrauenswürdige Nicht-Ärzte erlaubt - §5 Abs.3 der Landesverordnung über das Leichenwesen vom 30.11.1995, GVBl. S. 395.

25 DDR-GBl. 1979, S. 4; siehe auch Wegener, Zur Rechtslage und Praxis der Obduktionen in der früheren DDR, MedR. 1991, S. $231 \mathrm{ff}$. 
automatisch stattfindenden Verwaltungssektion bei »nicht-natürlichem Tod« (inklusive Verdacht) und »nicht-aufgeklärter« Todesart, insbesondere bei Todesfällen im Zusammenhang mit medizinischen Maßnahmen, aber auch bei allein aus medizinischer Sicht unklaren Todesfällen. Im Jahre 1990 versuchte eine Arbeitsgruppe der Gesundheitsministerkonferenz der Länder eine »Verbesserung der ärztlichen Leichen$s c h a u$ « durch Erarbeitung eines ländereinheitlichen Leichenschauscheines. ${ }^{26}$ Ebenfalls 1990 veranstaltete die »Deutsche Gesellschaft für Medizinrecht (DGMR) e.V.« den IV. Einbecker Workshop vom 13. bis 14. Oktober 1990. Dort anwesende Mediziner und Juristen erhoben die Forderung nach einer gesetzlichen Regelung des Obduktionsrechts. ${ }^{27}$ Im Mai des gleichen Jahres forderte auch der 93. Deutsche Ärztetag ein Obduktionsgesetz auf der Grundlage einer indikativen Lösung. ${ }^{28}$ Seither sind in einer Reihe von Bundesländern (so in Bremen, Berlin, Hamburg, NRW, Saarland, Sachsen, Mecklenburg-Vorpommern, Thüringen) neue Friedhofs- und Bestattungsgesetze verabschiedet worden, die nunmehr weitergehende Regelungen des Obduktionsrechtes, insbesondere Regelungen für sog. klinische Sektionen enthalten. Dennoch sind die Regelungsdichte und die geregelten Tatbestände weiterhin äußert uneinheitlich.

\section{Die gegenwärtige Situation des Leichenschauwesens}

Im Grundsatz sehen alle landesrechtlichen Vorschriften bei jedem Sterbefall eine sorgfältige äußere Leichenschau vor. ${ }^{29}$ Diese Leichenschau ist von einem Arzt vorzunehmen. Der jeweils zuständige Landesgesetzgeber geht davon aus, daß alle Ärzte über die erforderlichen Kenntnisse und Erfahrungen verfügen, um die äußere Leichenschau vornehmen und um den eingetretenen Tod zweifelsfrei feststellen zu können. Forderungen, jede Leichenschau von einem neutralen, erfahrenen Arzt durchführen zu lassen, konnten sich nicht durchsetzen. Jede Leichenschau beendet der Arzt mit der Ausstellung einer Todesbescheinigung. Für jene Fälle, in denen eine gründliche Leichenschau nicht möglich ist (zum Beispiel beim Einsatz der Notärzte im Rettungsdienst) ist teilweise die Ausstellung eines vorläufigen Totenscheines vorgesehen. ${ }^{30}$ Die Todesbescheinigung untergliedert sich in einen vertraulichen und einen nicht-vertraulichen Teil, sie ist auch für Totgeborene oder in der Geburt verstorbene Leibesfrüchte mit einem Gewicht von wenigstens $500 \mathrm{~g}$ auszufüllen. Dass jeder Arzt ohne weitere Kontrolle seiner Erfahrung und Qualifikation eine Leichenschau durchführen darf und muss, gilt als eine der Ursachen für die unzureichende Erfassung nichtnatürlicher Todesfälle bei der ärztlichen Leichenschau. ${ }^{31}$

26 Siehe Schreiben des federführenden Ministeriums des Landes Schleswig-Holstein vom 26. März 1990 an die Ärztekammern, in: Wagner, Ärztliche Leichenschau, Dtsch Ärztebl (1990) Heft 44, S. 2018 ff.

27 Empfehlungen der DGMR e.V., MedR. 1991, S. 76.

28 93. Deutscher Ärztetag: Entschließungen zum Tagesordnungspunkt V - Obduktionsgesetz. Dtsch Ärztebl 87 (1990), S. 1121 (1136).

29 Ausführlich: Madea/Dettmeyer, Ärztliche Leichenschau und Todesbescheinigung, Dtsch Ärztebl 100 (2003), S. 2458-2473.

30 Zuletzt z.B. gem. § 6 Abs. 1 thüring. BestG. 


\section{Der nichtvertrauliche Teil der Todesbescheinigung}

Der nichtvertrauliche Teil ist für das zuständige Standesamt und enthält Angaben zur Person des Verstorbenen ${ }^{32}$, zur Art der Identifikation, zur Feststellung des Todes, zur Todesart sowie Zusatzangaben bei Totgeborenen und Hinweise zum Infektionsschutzgesetz. Unter der Rubrik »Todesart « ist anzukreuzen:

»Natürlicher Tod«, »Nicht-natürlicher Tod« oder (nicht in allen Bundesländern) »Ungeklärt, ob natürlicher oder nicht-natürlicher Tod; Obduktion erforderlich«. Ein »natürlicher Tod « ist ein solcher aus (vorbestehender) krankhafter innerer Ursache.

Auf eine Legaldefinition des nichtnatürlichen Todes verzichten die meisten landesgesetzlichen Regelungen, jedoch heißt es nunmehr in $\S 6$ Abs. 3 S.2 thüring. BestG: »Als nicht natürlich ist ein Tod anzunehmen, der durch Selbsttötung, einen Unfall, einen ärztlichen Behandlungsfehler oder durch eine sonstige äußere Einwirkung, bei der ein Verhalten eines Dritten ursächlich gewesen sein könnte (Tod durch fremde Hand), eingetreten ist. «Die Feststellung eines »natürlichen Todes« bleibt ohne weitere Konsequenzen, der Leichnam kann unmittelbar bestattet werden (soweit nicht eine See- oder Feuerbestattung vorgesehen ist). Die Feststellung eines »nichtnatürlichen Todes « führt automatisch zur Einschaltung der Ermittlungsbehörden (Polizei, Staatsanwaltschaft), diese können gegebenenfalls nach Maßgabe der Vorschriften der Strafprozeßordnung weitere Maßnahmen einleiten. Landesgesetzliche Vorschriften zur Durchführung der ärztlichen Leichenschau sind unterschiedlich gestaltet, auf bekannte Mißstände bei der ärztlichen Leichenschau wurde insofern reagiert, als z.B. das saarländ. BestG in $\S 15$ Abs. 1 ebenso wie das nordrhein-westf. BestG in $\S 9$ Abs. 3 im Gegensatz zu früher nun die Verpflichtung festschreiben, dass der Arzt den Leichnam in unbekleidetem Zustand gründlich zu untersuchen hat. Kann der Leichenschauarzt dabei die Todesart nicht klären, so soll er die Rubrik »Todesursache ungeklärt« ankreuzen, zugleich erklärt er damit, dass er eine Obduktion für erforderlich hält. Auch in diesem Fall werden die Ermittlungsbehörden tätig. Sollten diese anschließend zu dem Ergebnis kommen, dass auf der Grundlage des Strafprozeßrechts mangels hinreichender Anhaltspunkte für einen (fremdverschuldeten) nichtnatürlichen Tod im Sinne des $\S 159$ StPO eine Obduktion nicht erforderlich ist, so kommt es in der Regel zur Bestattung des Leichnams. Die Todesursache bleibt in diesem Fall ungeklärt! Eine gesetzliche Ermächtigungsgrundlage zur Anordnung einer Obduktion (also eine Verwaltungssektion) in den Fällen derartiger medizinisch unklarer Todesfälle gibt es derzeit bundesweit nicht (dem nahe kommende Regelungen für eine außergerichtliche Sektion bestehen in Bremen und Hamburg; s.u.).

Vereinzelt wird die Ansicht vertreten, dass die Staatsanwaltschaft berechtigt und entsprechend dem Legalitätsprinzip auch verpflichtet sei, im Rahmen einer sog. Leichensache gemäß $§ 159,87$ Abs.4 StPO i.V.m. Nr.33 f. der Richtlinien für das Straf-

31 Vennemann/Du Chesne/Brinkmann, Die Praxis der ärztlichen Leichenschau, Dtsch Med Wschr 126 (2001), S. 712-716.

32 Insbesondere die zur Eintragung in das Sterbebuch und für die Bestattung erheblichen Angaben, vergl. § 37 PStG vom 8. August 1957 i.d.F. vom 17. Juli 1970, BGB1.I, S. 1099. 
verfahren und das Bußgeldverfahren (RiStBV) Leichenöffnungen zu beantragen, wenn ein »nichtnatürlicher« Tod nicht von vorneherein völlig ausgeschlossen werden kann, und »eine Obduktion geeignet ist, tatsächliche Anhaltspunkte für oder gegen eine insoweit strafbare Handlung zutage zu fördern. ${ }^{33}$ Aus dieser recht weiten Interpretation der genannten Vorschriften der StPO wird dann gefolgert, dass es daneben der Einführung einer Verwaltungssektion nicht bedürfe ${ }^{34}$, die Leichenöffnung im Rahmen der Untersuchung in Leichensachen gemäß § 159 StPO schließe alle Fälle ein, für die eine Verwaltungssektion gefordert werde. In allen Fällen unklarer Todesursache oder Todesart sei eine Vermutung bezüglich eines »nichtnatürlichen « Todes nicht von vorneherein völlig ausgeschlossen. ${ }^{35}$ Würde diese Beurteilung zutreffen, so dürfte es Fälle von beschlagnahmten aber später ohne Obduktion freigegebenen Leichen, bei denen die Todesursache nicht bekannt ist, bei einer »ermessensgerechten Entscheidung « der Staatsanwaltschaft nicht geben. ${ }^{36}$ Mag diese juristische Bewertung der Vorschriften der Strafprozeßordnung vertretbar sein, so zeugt sie doch von einer krassen Unkenntnis der tatsächlichen Interpretation der $\S \S 159,87$ ff. StPO durch die Staatsanwaltschaften. ${ }^{37}$ Bei Übernahme der dargelegten Ansicht durch die Staatsanwaltschaften müßten etwa alle Fälle eines plötzlichen Kindstodes auch ohne jeden Anhalt für ein Fremdverschulden obduziert werden, denn in diesen Fällen sind Todesart und Todesursache immer unklar. ${ }^{38}$ Tatsächlich lassen die Staatsanwaltschaften in solchen und zahlreichen ähnlichen Fällen trotz ungeklärter Todesursache gerade deshalb nicht obduzieren, weil sich ermittlungsseitig kein Anhalt für einen »nichtnatürlichen« Tod ergeben hat und damit die Vorschriften der Strafprozeßordnung als Rechtsgrundlage für eine Obduktion nicht mehr in Betracht kommen. Auch erfolgt - nach einer allerdings älteren Untersuchung - seitens der Staatsanwaltschaft statistisch gesehen eine Entscheidung zugunsten der Todesursachenklärung durch eine Obduktion nach Beschlagnahme der Leiche nur im Verhältnis 1:9 bis 1:19, d.h. nur jeder 10. bis 20. unklare Todesfall wird einer tatsächlichen medizinischen Todesursachenklärung zugeführt. ${ }^{39}$

33 Schweitzer, Zu Problemen von Leichenschau und Totenbescheinigung unter besonderer Berücksichtigung des Strafrechts, S. 98/99.

34 Schweitzer, S. 102.

35 Schweitzer, S. 102.

36 Schweitzer, S. 102.

37 Diese vertreten wenigstens teilweise die Ansicht, eine Obduktion könne nur erfolgen, wenn ermittlungsseitig ein »durch tatsächliche Anhaltspunkte konkretisierter Verdacht einer strafbaren Handlung vorliegt«, vgl. Maiwald, Zur Ermittlungspflicht des Staatsanwalts in Todesfällen, NJW 1978, S. 561 (564).

38 Zur Behinderung gerade der Erforschung des plötzlichen Kindstodes mangels einer forensischen Indikation zur Obduktion: Albani, Der plötzliche Säuglingstod: Eine absolute Obduktionsindikation, MedR. 1991, S. 243 ff., mit dem ausdrücklichen Hinweis auf die fehlende Möglichkeit einer Verwaltungsobduktion.

39 Brinkmann/Kleiber/Janssen, Pathologe 2 (1981), S. 201 (203). 


\section{Der vertrauliche Teil der Todesbescheinigung}

Der vertrauliche Teil der Todesbescheinigung ist für das Gesundheitsamt bestimmt. Dabei sind anzuführen der zuletzt behandelnde Arzt und welche sicheren Zeichen des Todes bei der äußeren Leichenschau anzutreffen waren, gegebenenfalls ob eine Reanimationsbehandlung (Wiederbelegungsmaßnahmen) erfolgte und über welchen Zeitraum im Elektrokardiogramm (EKG) keine Herztätigkeit mehr feststellbar war. Ferner sind Angaben zur Todesursache zu machen. Dort ist anzugeben, welche Krankheit oder Verletzung den Tod unmittelbar herbeigeführt hat und welche Krankheiten oder Verletzungen dem zugrunde lagen. Weiterhin ist die Frage zu beantworten, welche anderen Krankheiten für den Tod mitursächlich waren. Diese Daten bilden die Grundlage für die von den Gesundheitsämtern geführten Statistiken, insbesondere für die Todesursachenstatistik. ${ }^{40}$ Sollte eine Obduktion durchgeführt worden sein, so sind die gleichen Fragen in Kenntnis des Obduktionsbefundes zu beantworten. Die Obduktion ermöglicht in diesem Falle zugleich unmittelbar die Überprüfung der vom Leichenschauarzt zuvor auf dem Formular angegebenen Diagnosen/Befunde zur Todesart und zur Todesursache. In einer weiteren Rubrik sind Angaben möglich bei Unfall, Vergiftung, Gewalteinwirkung und Selbsttötung. Die Unfallkategorie soll näher spezifiziert werden, bei Kindern unter $1 \mathrm{Jahr}$ sollten weitere Angaben zum Geburtsort, zu Länge und Gewicht des Kindes, zur Frage der Mehrlingsgeburt und zur Lebensdauer gemacht werden. Todesfälle im Zusammenhang mit einer Schwangerschaft sowie bis 6 Wochen nach der Entbindung werden gesondert erfaßt.

\section{Kritik der gegenwärtigen Praxis der Leichenschau}

Die Kritik an der Praxis der Leichenschau in allen Bundesländern setzt an bei der Qualität der ärztlichen Leichenschau und bei den Folgen für die Todesursachenstatistik. In allen Bundesländern wird die Leichenschau durch einen approbierten Arzt vorgenommen. Dieser trägt allein die Verantwortung für die Durchführung und Dokumentation der Leichenschau. Voraussetzung einer sorgfältigen ärztlichen Leichenschau ist die Untersuchung des vollständig entkleideten Leichnams »von allen Seiten und im Bereich aller Körperöffnungen «. ${ }^{41}$ Das Unterlassen der Entkleidung bei der Leichenbesichtigung und nachfolgend die fälschliche Angabe eines »natürlichen« Todes kann schwerwiegende Folgen haben.

Dazu einige Beispiele: Übersehen wurden die kirschroten Leichenflecke als Zeichen einer tödlichen Kohlenmonoxid-Vergiftung ${ }^{42}$, eine tödliche Herzschußverletzung, zahlreiche Messerstiche im Rücken, Kopfschußverletzungen u.a.m. ${ }^{43}$ Zahlreiche Einzelfälle und Studien haben einen hohen Anteil an übersehenen oder falsch interpretier-

40 Zur Qualität der Todesursachenstatistik zuletzt: Schubert-Fritschle/Eckel/Eisenmenger/ Hölzel, Qualität der Angaben von Todesbescheinigungen, Dtsch Ärztebl 99 (2002) S. 5055. Zum Einsichtsrecht in den vertraulichen Teil der Todesbescheinigung: Dettmeyer/ Madea, Ärztliches Schweigerecht bezüglich Daten der Leichenschau, NStZ 1999, S. 605.

41 Brinkmann/Püschel, Definition natürlicher, unnatürlicher, unklarer Tod, MedR. 1991, S. 233 ff.

42 AG Wennigsen, NJW 1989, S. 786.

43 Brinkmann/Püschel, MedR. 1991, S. 233 (235) m.w.N. 
ten Befunden am menschlichen Leichnam ergeben. ${ }^{44}$ Eine ältere Statistik mit 13551 Fällen wies einen Anteil von 62\% Fehldiagnosen bei der ärztlichen Leichenschau auf, darunter immerhin $6 \%$ mit verkanntem Fremdverschulden. ${ }^{45}$ In Extremfällen wurden gar Todesbescheinigungen für noch Lebende ausgestellt. ${ }^{46}$ Häufig ergeben sich Schwierigkeiten bei der Einteilung in die drei Kategorien »natürlicher«, »nichtnatürlicher « und »unklarer« Tod. Nach einer (älteren) Studie in Niedersachsen an 315 Krankenhäusern bescheinigen ca. 6\% aller Krankenhausärzte in der Todesbescheinigung grundsätzlich, d.h. bei jedem Todesfall, einen natürlichen Tod. ${ }^{47}$ Bei »nichtnatürlichem« Tod - Gewalteinwirkung, Vergiftung, Suizid, Tod infolge ärztlichen Eingriffs, Verkehrsunfall, ärztlicher Behandlungsfehler etc. - wird in bis zu 30\% der Fälle ein »natürlicher « Tod auf der Todesbescheinigung angegeben. ${ }^{48}$ Zunehmende Schwierigkeiten zeigten sich nach allen Untersuchungen, wenn zwischen dem Ereignis und dem Todesfall eine kompliziertere Kausalkette lag. Bei Suizid-Fällen etwa ergab sich ein Dunkelfeld von nahezu $100 \%{ }^{49}$, bei Verkehrsunfällen von bis zu $20 \%{ }^{50}$, in denen die Todesart unzutreffend als natürlich bezeichnet wurde. Insofern verwundert die Feststellung nicht, daß in der Klassifikation der Todesart (»natürlich«, »nicht-natürlich«, »unklar «), wie sie derzeit in der Bundesrepublik Deutschland erfolgt, grobe und offensichtlich systematische Fehler vorkommen. ${ }^{51}$ Ein Rückschluß auf die tatsächliche Häufigkeit »nichtnatürlicher« Todesfälle ist angesichts der fehlerhaften Klassifikation der Todesart kaum möglich.

Als Ursachen für diesen Zustand werden mehrere Gründe genannt: mangelnde Leichenschauerfahrung vieler Ärzte, der verdrängend wirkende Wunsch des Arztes, den Angehörigen eine Obduktion zu ersparen, die schwere Überschaubarkeit kausaler Zusammenhänge sowie Probleme in der Diagnostik, wenn die Todesursache nach dem ICD-Code ${ }^{52}$ klassifiziert werden muß. ${ }^{53}$ Die Tatsache, daß der Arzt im Falle eines

44 Vergl. dazu nur: Metter, Ärztliche Leichenschau und Dunkelziffer bei unnatürlichen Todesfällen, Kriminalistik 1978, S. 155; Mätzler, Ärztliche Todesbescheinigungen für Lebende, Kriminalistik 1978, S. 157 ff.; Trube-Becker, Leichenschauschein und Todesursachenstatistik, VersMed. 43 (1991), S. 37 ff.; Wagner, Das Verhalten des Arztes bei tödlichen Verkehrsunfällen, DÄ 66 (1969), S. 2748 ff.; Schleyer, Aktuelle Fragen der Leichenschau und Leichenöffnung, Dt. Zschr. ges. gerichtl. Med. 62 (1968), S. 55 ff.; Eisenmenger/Spann/Liebhardt, Bestattungsgesetze und Praxis der Leichenschau - eine kritische Bestandsaufnahme. Beitr. gerichtl. Med. 40 (1982), S. 49 ff.; Oehmichen/Saternus, Leichenschau und Todesbescheinigung, Kriminalistik 39 (1985), S. 2 ff.; Vock, Überprüfung von ca. 7000 Todesbescheinigungen. Konsequenzen für die ärztliche Aus- und Fortbildung. Beitr. gerichtl. Med. 42 (1984), S. 355 ff.

45 Schleyer, Dt. Zschr. ges. gerichtl. Med. 62 (1968), S. 55 ff.

46 Mätzler, Kriminalistik 1978, S. 157 ff.

47 Berg/Ditt, Problem der ärztlichen Leichenschau im Krankenhausbereich. Nds. Ärzteblatt 8 (1984), S. $162 \mathrm{ff}$.

48 Berg/Ditt, Nds. Ärzteblatt 8 (1984), S. 162 ff.

49 Rehm, Selbstmordstatistik der Stadt Münster/Westf., Untersuchungen über die Jahre 19711981, Med. Diss. Münster 1985.

50 Püschel/Brinkmann, Zur Dunkelziffer von Verkehrstoten, Hamb. Ärztebl. 38 (1984), S. 162 ff.

51 Metter, Kriminalistik 1978, $155 \mathrm{ff}$.

52 ICD-Code der WHO = International Classification of Diseases.

53 Wegener, MedR. 1991, S. 231. 
nicht korrekten Ausfüllens des Totenscheines jedenfalls dann mit einem Ordnungswidrigkeitenverfahren rechnen muß, wenn spätere Ermittlungen einen »nichtnatürlichen « Tod, womöglich mit Fremdverschulden, beweisen, hat an dem beschriebenen Zustand nichts geändert. Immerhin wurden in den Jahren 1980 bis 1984 in Berlin in 174 Fällen derartige Ordnungswidrigkeitenverfahren eingeleitet. ${ }^{54}$ Schließlich sei erwähnt, daß bei der schlechten Qualität der ärztlichen Leichenschau gelegentlich auch forensisch relevante Befunde einschließlich übersehenen Fremdverschuldens vorkommen. ${ }^{55} \mathrm{Im}$ Hinblick auf forensische Fragestellungen forderte u.a. der Bund Deutscher Kriminalbeamter »den amtlich bestellten Leichenschauarzt mit qualifizierten gerichtsmedizinischen Kenntnissen ${ }^{56}$

Zur Validität der Todesursachenstatistik gibt es eine Reihe von Studien. So wurden in ca. 35\% der Fälle im Bereich der Chirurgie und der Inneren Medizin im Rahmen klinischer Obduktionen abweichende Todesursachen gefunden ${ }^{57}$ Eine sorgfältige größere Studie ergab, daß zwischen dem auf der Todesbescheinigung angegebenen und dem durch die Obduktion ermittelten Grundleiden in nahezu 40\% der Fälle keine Übereinstimmung bestanden hat (wobei nach Schätzungen wohl »nur« $10 \%$ bis $30 \%$ der Patienten bei richtiger Diagnose anders und erfolgreicher hätten behandelt werden können ${ }^{58}$ ). Ähnliche Resultate fanden sich bei größeren ausländischen Studien. ${ }^{59}$ Auf die wichtige Kontrollfunktion der Obduktionen für eine aussagekräftige Todesursachenstatistik wurde in der Vergangenheit häufig hingewiesen. Dennoch setzte sich der Trend zu sinkenden Obduktionszahlen in den letzten Jahren fort, seit 1989 auch in der Ländern der ehemaligen DDR. ${ }^{60}$

54 Schneider, Die Leichenschau, S. 15.

55 Diese Tatsache dürfte wesentlich zu folgender Feststellung beigetragen haben: »Nach fast 40jähriger Tätigkeit als Rechtsmediziner kann ich jedenfalls ohne schlechtes Gewissen die Behauptung aufstellen, daß der »perfekte Mord « in seinen vielfältigen Formen ein alltägliches Ereignis ist«, so Trube-Becker, VersMed. 43 (1991), S. 37 (40).

56 Schneider, Die Leichenschau, S. 18.

57 Püschel/Kappus/Janssen, Ärztliche Leichenschau im Krankenhaus. Arzt und Krankenhaus 60 (1987), S. $101 \mathrm{ff}$.

58 Modelmog/Goertchen/Steinhard/Sinn/Stahr, Vergleich der Mortalitätsstatistik einer Stadt bei unterschiedlicher Obduktionsquote (Görlitzer Studie), Pathologe 12 (1991), S. 191 ff.; siehe auch Grundmann, Autopsy as clinical quality control: a study of 15143 autopsy cases, in vivo 8 (1994), S. $945 \mathrm{ff}$.

59 Vgl. Sarode et al., Autopsy findings and clinical diagnoses: a review of 1000 cases, Hum. Pathol. 24 (1993), S. 194 ff.; Kircher et al, The autopsy as a measure of accuracy of the death certificate, N.Engl.J.Med. 313 (1985), S. 1263 ff.; Stevanovic et al, Correlation of clinical diagnoses with autopsy findings: a retrospective study of 2145 consecutive autopsies, Hum. Pathol. 17 (1986), S. 1225 ff.; Battle et al, Factors influencing discrepancies between premortem and postmortem diagnoses, JAMA 258 (1987), S. 339 ff.

60 Dazu beispielhaft Habeck/Waller, Die Anzahl der Obduktionen im Institut für Pathologie Chemnitz vor und nach der Wiedervereinigung (1987-1992), Pathologe 14 (1993), S. 188 ff. 


\section{Die gegenwärtige Situation im Obduktionsrecht}

Die zuständigen Behörden können derzeit auf bundesgesetzlicher Grundlage eine Obduktion anordnen

a) gemäß $\S 87 \mathrm{ff}$. StPO (strafprozessuale bzw. gerichtliche Sektion)

b) gemäß $§ 16$ IfschG (frühere sog. Seuchensektion)

c) gemäß $\S 3$ Abs.2 Ziff.2 FeuerbestG (Feuerbestattungssektion; soweit noch in Kraft)

d) gemäß §§ 103 ff. SGB VII (sozialversicherungsrechtliche Obduktion).

Mittlerweile teilweise landesgesetzlich geregelte Obduktionen finden statt in den Instituten für Pathologie und Anatomie der Krankenhäuser und Universitäten:

a) die klinisch-wissenschaftliche Sektion

b) die anatomische Obduktion

c) die privatversicherungsrechtlich begründete Sektion

d) Obduktionen im Auftrag der Totensorgeberechtigten (sog. Privatsektion).

\section{Die klinisch-wissenschaftliche Obduktion (klinische Sektion)}

Die klinische Sektion findet nahezu ausschließlich in den Instituten für Pathologie der Universitäten und Krankenhäuser statt. Rechtliche Grundlage sind - soweit gesetzliche Regelungen fehlen - die Allgemeinen Geschäftsbedingungen (AGB) in Krankenhausaufnahmeverträgen. Diese enthalten eine sog. »Sektionsklausel«. Deren Zulässigkeit wurde im Rahmen einer Verbandsklage nach § 13 Abs.2 Nr.1 AGBGesetz vom BGH grundsätzlich bejaht. ${ }^{61}$ Derartige »Sektionsklauseln « lassen eine Obduktion zu, wenn der Verstorbene zu Lebzeiten eingewilligt hat oder wenn der erreichbare nächste Angehörige zustimmt und dem Krankenhausarzt ein entgegenstehender Wille des Verstorbenen nicht bekannt geworden ist. ${ }^{62}$ Entgegen der Auffassung mancher Kommentare - etwa seitens des Berufsverbandes Deutscher Pathologen e.V. ${ }^{63}$ - war jedoch das eigentliche juristische Problem, nämlich die Frage nach dem Überraschungscharakter ( 33 AGB-Gesetz) einer »Sektionsklausel« in einem Krankenhausaufnahmevertrag nicht Gegenstand der Entscheidung des BGH. Juristisch ist die Situation daher weiterhin unsicher ${ }^{64}$, faktisch dürfte das Urteil trotzdem zur

61 BGH, JZ 1990, 923 ff. mit sehr kritischer Anmerkung von Ackmann; zuvor OLG Koblenz, NJW 1989, 2950 ff.; s. auch Gounalakis, Formularmäßige ärztliche Aufklärung im Lichte des AGB-Gesetzes, NJW 1990, S. 752; Meister, Zur Zulässigkeit von Obduktions- und Transplantationsklauseln in Allgemeinen Vertagsbedingungen (AVB) für Krankenhäuser, Das Krankenhaus 2000, S. 302-308.

62 Vgl. BGHZ 9, 145 (149); OLG München, NJW 1976, 1805; 2294 (2296); Haas, L., NJW 1988, S. 2929 (2932 ff.); Bunte, NJW 1986, S. 2351 (2354); Becker, W., JR 1951, S. 328 (331);

63 Insbesondere kann nicht, wie der Berufsverband Deutscher Pathologen e.V. in einem Schreiben vom 27.8.1990 wohl angenommen hat, von einer durch den BGH erfolgten Billigung der sogenannten »Widerspruchslösung « ausgegangen werden, dazu insbesondere Solbach, Zur Frage der Zulässigkeit vorformulierter Einwilligungserklärungen für eine Sektion in Krankenhausaufnahmeverträgen, MedR. 1991, S. 27 ff.

64 Anm. Deutsch, NJW 1990, S. 2315 zum BGH-Urteil vom 31.5.1990, NJW 1990, 2313; ebenso Solbach, MedR. 1991, S. 27 ff. 
Rechtssicherheit beigetragen haben. ${ }^{65}$ Teilweise gilt auch die Regelung, daß eine Obduktion durchgeführt werden kann, wenn bei fehlender eindeutiger Erklärung des Verstorbenen die nächsten Angehörigen nicht innerhalb einer gesetzten Frist (z.B. 24 Std.) nach Information über den Todesfall Widerspruch erheben. ${ }^{66}$ Mehrheitlich wird davon ausgegangen, daß eine klinische Sektion gegen den Willen des Verstorbenen rechtswidrig ist. ${ }^{67}$ Bei fehlender Willensäußerung des Verstorbenen soll die Zustimmung der totensorgeberechtigten Hinterbliebenen erforderlich sein, im Einzelfall soll auch ein Rückgriff auf eine mutmaßliche Einwilligung des Verstorbenen in Betracht kommen. ${ }^{6}$

\section{Die anatomische Obduktion}

Unter den Begriff der anatomischen Obduktion werden jene Obduktionen gefaßt, die im Rahmen der studentischen Ausbildung sowie für wissenschaftliche Fragestellungen an den Universitäten stattfinden. ${ }^{69}$ Dabei handelt es sich um die über mehrere Monate verlaufende sorgfältige Präparation der anatomischen Strukturen des menschlichen Leichnams zum Zwecke der Ausbildung angehender Mediziner sowie für wissenschaftliche Fragestellungen. In der Regel liegt bei den für anatomische Obduktionen verwendeten Leichen eine zu Lebzeiten erteilte Einwilligung des Betroffenen vor, eine solche Einwilligung wird angesichts der auch gegenüber »normalen« Obduktionen ungewöhnlich langen und sehr weitreichenden Präparation auch in der Literatur verlangt. ${ }^{70}$ Früher war die anatomische Sektion nur teilweise gesetzlich oder durch behördliche Erlasse geregelt ${ }^{71}$, mittlerweile existieren einige landesgesetzliche Regelungen.

65 Ehlers, Die Sektion zwischen individuell erklärter Einwilligung und Allgemeinen Geschäftsbedingungen in Krankenhausaufnahmeverträgen, MedR. 1991, S. 227 ff.; auf die Notwendigkeit einer Vermeidung des Überraschungscharakters einer »Sektionsklausel « weisen auch die Empfehlungen der DGMR e.V. ausdrücklich hin, MedR. 1991, S. 76.

66 Dazu Künzel, Zur rechtlichen Beurteilung einer in Krankenhausbedingungen verwendeten Sektionsklausel, die eine Widerspruchslösung vorsieht, Pathologe 11 (1990), S. 65 ff.; ders., Zur Zulässigkeit vorformulierter Einwilligungserklärungen für eine innere Leichenschau (Sektionseinwilligung) in Krankenhausaufnahmeverträgen, Pathologe 11 (1990), 319 ff.

67 Ehlers, MedR. 1991, S. 227 (228); Mehrhoff/Müller, MedR. 1990, S. 125 (126); Trockel, Die Rechtswidrigkeit klinischer Sektionen, S.148 ff.; Becker, JR 1951, S. 328 (331); a.A., u.a. mit dem Argument, bei gesetzlichen Obduktionen komme es auch nicht auf den Willen des Verstorbenen an: Zimmermann, NJW 1979, S. 569 (573 f.); Laufs, Arztrecht, Rdnr.268; Heifer/Pluisch, Rechtsmedizin 1991, S. 73 (76) gehen, allerdings ohne Berücksichtigung verfassungsrechtlicher Aspekte, bereits von der Zulässigkeit klinischer Sektionen aus, wenn ein Widerspruch nicht vorliegt.

68 Hirsch/Schmidt-Didczuhn, S. 28; Mehrhoff/Müller, MedR. 1990, S. 125 (127).

69 Eine Definition des Begriffes »anatomische Sektion« enthält § 6 Abs.1 des Entwurfes der Berliner CDU-Fraktion vom 22.6.1973 (Drs. 6/948): »Die anatomische Sektion ist die Zergliederung von Leichen oder Leichenteilen zum Zwecke der Forschung oder Lehre über den Aufbau des menschlichen Körpers."

70 Franzki, MedR. 1991, S. 223 (226).

71 Vgl. GuVOBl. Kgr. Sachsen 1912, S. 465; Erl. d. SozM BW v. 16.5.1974, GVBl. S.656; RdErl. d. nds. SozM v. 8.8.1962, nds. MBl. 1962, S. 280. 


\section{Die privatversicherungsrechtlich begründete Obduktion}

Von privaten Versicherungsträgern werden ebenso wie von den Berufsgenossenschaften Obduktionen zur Frage eines Ursachenzusammenhangs zwischen dem eingetretenen Tod und der versicherten Tätigkeit in Auftrag gegeben. Gelegentlich erfolgen zum Zwecke der Beurteilung versicherungsrechtlich relevanter Kausalzusammenhänge auch Exhumierungen.$^{72}$ Rechtsgrundlage sind die privaten Versicherungsverträge. Die potentiell Begünstigten haben die Möglichkeit, der geplanten Obduktion zu widersprechen, jedoch mit nachteiligen Konsequenzen hinsichtlich der Beweislage. ${ }^{73}$

\section{Privatsektionen}

Relativ selten werden Pathologen oder Rechtsmediziner von privater Seite mit der Durchführung einer Obduktion und der Erstellung eines Gutachtens beauftragt. Dies kann der Fall sein, wenn der Verstorbene zu Lebzeiten eine Obduktion seines Leichnams bestimmt hat oder wenn - häufiger - die totensorgeberechtigten Hinterbliebenen eine innere Leichenschau wünschen. Letzteres in der Regel zur Absicherung der Beweisführung versicherungsrechtlich begründbarer Ansprüche oder im Rahmen eines Arzthaftungsprozesses. ${ }^{74}$

In der Bundesrepublik Deutschland - alte Bundesländer, seit 1989 auch neue Bundesländer - ist die Obduktionsfrequenz seit Jahren stark rückläufig. ${ }^{75}$

Mit einer niedrigen Obduktionsquote ist, angesichts der Fehlerquote der bloßen äußeren Leichenschau (siehe oben), eine zuverlässige Todesursachenstatistik (Mortalitätsstatistik) und Krankheitsstatistik (Morbiditätsstatistik) nicht mehr möglich. Auch kann die Frage nicht ausbleiben, was teurer ist: »die falschen Maßnahmen, die aufgrund einer unzuverlässigen Todesursachenstatistik ergriffen werden, oder die mit der ....Verwaltungssektion verbundenen Mehrkosten? « ${ }^{76}$ Der seit vielen Jahren immer wieder aufgekommene Ruf nach einer gesetzlichen Regelung des Obduktionswesens reicht daher bis in die jüngste Zeit. ${ }^{77}$ Weitere denkbare Eingriffe in Rechte des Ver-

72 Vgl. Naeve/Bandmann, Über Fragestellungen, Ergebnisse und Aussagewert versicherungsmedizinischer Obduktionen nach Exhumation, Lebensversicherungsmedizin 1981, S. 37 ff.

73 BGH, VersR. 1991, 1365.

74 Vgl. Jansen, Unterbliebene Sektion und Umkehr der Beweislast im Arzthaftpflichtprozeß, Pathologe 14 (1993), S. 68 ff.

75 Nach einer Hochrechnung empirisch ermittelter Angaben auf der Basis von Umfragen, an denen sich etwa die Hälfte der Pathologischen Institute in der Bundesrepublik Deutschland beteiligt hat, ist lt. Berufsverband Deutscher Pathologen e.V. von folgenden Obduktionszahlen auszugehen: 1982 (65.300), 1983 (64.400), 1984 (63.000), 1985 (54.800), 1986 (49.300), 1987 (49.700), 1988 (47.500), 1989 (51.750), 1993 (einschl. der neuen Bundesländer: 47.880); unklar ist, ob diese Zahlen auch die in den gerichtsmedizinischen Instituten durchgeführten Obduktionen erfassen; beispielhaft zum Rückgang der Obduktionszahlen in den neuen Bundesländern Habeck/Waller, Pathologe 14 (1993), S. 188 ff.

76 Wagner, Ärztliche Leichenschau, Dtsch Ärztebl 87 (1990), S. 2018 ff.

77 Vgl. nur Naeve, Über ärztliche Bemühungen um gesetzliche Einführung von Verwaltungssektionen in Deutschland. Literaturüberblick 1895-1979, Öff. Gesundh.-Wesen 42 (1980), S. 191 ff.; Klinge, Todesbegriff, Totenschutz und Verfassung, S. 83; Dettmeyer/Schmidt/ Madea, Rechtsgrundlagen und Ermessensspielräume bei der amtsärztlich angeordneten 
storbenen beziehungsweise des Leichnams seien hier nur erwähnt: Versuche mit Leichen, etwa im Rahmen wissenschaftlicher Fragestellungen bei sog. Crash-Tests ${ }^{78}$, Schießversuche ${ }^{79}$ oder die postmortale Verwendung von dem Leichnam entnommenen Organen und Geweben einschließlich der Weitergabe an interessierte Dritte. ${ }^{80}$ Eine solche Entnahme von Organen oder auch Organteilen findet statt zum Zwecke der Wiederverwendung zugunsten Lebender. So besteht etwa Interesse an der harten Hirnhaut $^{81}$, an den Gehörknöchelchen, an der Hirnanhangsdrüse etc. Derartige Entnahmen dienen nicht der Klärung der Todesursache und sind insofern auch nicht notwendiger Bestandteil einer Obduktion. Solche Entnahmen bedürfen einer gesonderten Betrachtung und gesetzlichen Regelung. ${ }^{82}$ In einzelnen jüngeren landesgesetzlichen Regelungen finden sich ansatzweise Bestimmungen zur autoptischen Entnahme von Organen und Gewebe. ${ }^{83}$ Gerade die Einbehaltung von Organen und Geweben zum Zwecke der Weitergabe an Dritte - etwa die therapeutische Gewebeentnahme - ist landesgesetzlich (außer in Berlin) nicht geregelt. Die beschriebene unbefriedigende Situation im Leichenschau- und Obduktionsrecht hat zur verschiedenen Lösungsvorschlägen geführt, die im Leichenschaurecht von der exklusiven Durchführung der Leichenschau durch neutrale, erfahrene Ärzte bis zur gesetzlichen Konkretisierung der Art und Weise der Leichenschau (entkleideter Leichnam, Inspektion aller Körperöffnungen) reichen. Für das Obduktionsrecht werden verschiedene Lösungsmodelle diskutiert, um einerseits dem erforderlichen Regelungsbedarf gerecht zu werden, dem weiteren Sinken der Obduktionsquote entgegenzuwirken und andererseits grundrechtlich geschützte Interessen des Verstorbenen und seiner Hinterbliebenen hinreichend zu berücksichtigen.

Obduktion (Verwaltungssektion). Gesundheitswesen 62 (2000), S. 25-29.; Madea/Dettmeyer, Rechtsgrundlagen der Leichenschau, in: Madea (Hrsg.) Die ärztliche Leichenschau,

78 dazu Pluisch/Heifer, Die rechtliche Zulässigkeit von Leichenversuchen, NJW 1994, S. 2377 ff.

79 Dazu bereits Klee, DJZ 1910, S. 1403.

80 Vgl. Taupitz, JZ 1992, S. 1089 ff.

81 Der Verkauf von Hirnhäuten bei Entnahme aus ohnehin zur Vernichtung bestimmten Körperteilen nach einer Obduktion soll keine Störung der Totenruhe darstellen, Beschl. AG Berlin-Tiergarten, NStZ 1996, 544.

82 So werden in $\S 18$ Abs.3 des »Gesetzes über das Leichenwesen « der Freien Hansestadt Bremen die Voraussetzungen für die Entnahme von Leichenteilen festgelegt, zugleich bestimmt $\S 18$ Abs.4 ausdrücklich, entnommene Leichenteile »dürfen nicht Gegenstand von Rechtsgeschäften sein, die auf Gewinnerzielung gerichtet sind.«; vgl. aber Urteil des AG Berlin-Tiergarten vom 27.3.1996, NStZ 1996, 544; ausführlicher: Dettmeyer/Madea, Die postmortale Gewebeentnahme für Transplantationszwecke, Rechtsmedizin 12 (2002) S. 365-370; Dettmeyer/Madea, Aufklärung und Einwilligung bei der Beschaffung und Verarbeitung von menschlichen Zellen und Geweben, Rechtsmedizin 14 (2004) S. 85-93.

83 Explizit in $\S 10$ berl. Sektionsgesetz; in $\S 10$ Abs. $1 \mathrm{~S} .2$ des nordrhein-westf. BestG heißt es lediglich: «Die Obduktion umfasst auch die Entnahme von Organen und Gewebeteilen sowie deren Aufbewahrung «. Dagegen beschränken das ansonsten relativ ausführliche saarländ. BestG und das neue thüring. BestG die Entnahme von Organen und Geweben auf den Zweck der klinischen Sektion (§ 46 Abs.1 saarländ. BestG bzw. § 10 Abs.3 thüring. BestG, welches allerdings für den Fall der Zurückbehaltung von Organen eine entsprechende Information der Angehörigen verlangt). 


\section{Lösungsmodelle im Obduktionsrecht}

Während bei der Organtransplantation von einem hirntoten Spender auf einen Organempfänger gerade vorausgesetzt wird, daß eben »nur « die Hirnfunktionen erloschen sind - das Abwarten des Erlöschens sämtlicher Körperfunktionen würde das Ende der Transplantationsmedizin bedeuten - , ist die Situation im Obduktionsrecht eine andere. Während bei der Explantation von Organen Entscheidungen unter Umständen innerhalb von Stunden zu treffen sind, kann eine Obduktion in der Regel ohne Einbuße der Aussagekraft bei zwischenzeitlich gut gekühlt aufbewahrtem Leichnam auch noch nach Tagen erfolgen. Damit ist gegebenenfalls wertvolle Zeit gewonnen, um die häufig unbekannte Haltung des Verstorbenen und/oder seiner totensorgeberechtigten Hinterbliebenen zur Frage einer Obduktion der Leiche zu klären. Als Rechtsgrundlage für die Zulässigkeit insbesondere der klinischen Sektion werden dabei die aus der Diskussion zur Transplantationsproblematik bekannten Modelle diskutiert.

\section{Die enge und erweiterte Einwilligungslösung}

Bei der engen Einwilligungslösung muß der Verstorbene in die Obduktion seines Leichnams eingewilligt haben, Vorstellungen der Angehörigen werden nicht berücksichtigt. Bei der erweiterten Einwilligungslösung hat der Verstorbene in eine Obduktion nicht eingewilligt, es ist aber auch kein entgegenstehender Wille bekannt und eine Obduktion kann erfolgen, wenn die Totensorgeberechtigten eingewilligt haben. Das erweiterte Einwilligungsmodell entspricht im wesentlichen der derzeitigen Praxis bei der klinisch-wissenschaftlichen Sektion. Eine Obduktion ist danach zulässig, wenn primär die tatsächliche Einwilligung des Verstorbenen oder subsidiär die Einwilligung der Totensorgeberechtigten vorliegt und kein entgegenstehender Wille des Verstorbenen bekannt ist. Diese Regelung haben eine Reihe von Bundesländern übernommen (s.u.).

\section{Die enge und erweiterte Widerspruchslösung}

Nach der engen Widerspruchslösung darf eine Obduktion durchgeführt werden, wenn der Verstorbene zu Lebzeiten nicht widersprochen hat. Die Vorstellungen der Totensorgeberechtigten werden nicht berücksichtigt. Nach der erweiterten Widerspruchslösung können die Totensorgeberechtigten - soweit kein entgegenstehender Wille des Verstorbenen bekannt ist - der Obduktion innerhalb einer festgelegten Frist widersprechen. Bleibt der Widerspruch aus, so kann obduziert werden. Dieses Modell geht damit von einer Zustimmungsvermutung aus, das Schweigen des Verstorbenen zu Lebzeiten wird als Zustimmung gedeutet, ebenso gilt das Schweigen der Totensorgeberechtigten bis zum Ablauf der Widerspruchsfrist als Zustimmung. ${ }^{84}$ Die erweiterte

84 Als Beispiel sei auf die 1988 an der Medizinischen Hochschule Hannover geltenden Allgemeinen Vertragsbedingungen (AVB) hingewiesen, wo es unter dem Punkt »Innere Leichenschau « heißt: »Eine klinische Sektion (innere Leichenschau) wird durchgeführt, wenn der Leiter der Abteilung, in der der Kranke verstorben ist, oder der Direktor des Pathologischen 
Widerspruchslösung ist eher geeignet, die Obduktionsquote zu erhöhen als eine Regelung, die zwingend die positive Zustimmung der Hinterbliebenen verlangt. ${ }^{85}$

\section{Die Informationslösung}

Die sogenannte Informationslösung verlangt primär die Einwilligung des Verstorbenen in die Obduktion »seiner « Leiche. Fehlt diese Einwilligung und ist kein entgegenstehender Wille des Verstorbenen bekannt, so darf nach Information der Totensorgeberechtigten und Ablauf einer diesen einzuräumenden Widerspruchsfrist obduziert werden. Die Totensorgeberechtigten sind anläßlich der Information über die geplante Obduktion zugleich über ihr Widerspruchsrecht aufzuklären. Der Gesetzgeber soll präzisieren, was unter einer angemessenen Frist zu verstehen ist. Auch nach diesem Modell wird das bloße Schweigen sowohl des Verstorbenen als auch der Totensorgeberechtigten nach Fristablauf im Ergebnis als Zustimmung interpretiert. In der Praxis nähert sich daher die Informationslösung dem erweiterten Widerspruchsmodell. Die Notwendigkeit eines persönlichen ärztlichen Informationsgesprächs mit den totensorgeberechtigten Hinterbliebenen ermöglicht jedoch die ausführlichere Darlegung der Gründe, die im konkreten Fall aus medizinischer Sicht eine Obduktion als wünschenswert erscheinen lassen. Anschließend sollen die Totensorgeberechtigten in Kenntnis dieser Gründe nochmals innerhalb einer gesetzten Frist ihre Haltung überdenken können. Diese Regelung - Information der Angehörigen, Zulässigkeit der Obduktion bei fehlendem Widerspruch nach Ablauf einer Widerspruchsfrist - wurde ebenfalls von einigen Bundesländern übernommen (s.u.).

\section{Entwürfe und landesgesetzliche Regelungen im Obduktionsrecht}

In den letzten 35 Jahren wurden Regelungen für die (klinische) Sektion teils diskutiert, teils als Gesetzesvorlagen eingebracht, teils tatsächlich in Landesgesetze übernommen.

\section{Berliner Entwürfe von $1972 / 73$}

Im Jahre 1972 legte der Berliner Senat eine Vorlage für ein Bestattungsgesetz vor, welche Vorschriften zur klinischen und anatomischen Sektion enthielt. ${ }^{86}$ Dem antwor-

Instituts der Medizinischen Hochschule Hannover dieses unter angemessener Würdigung eventueller Bedenken des Ehegatten, der Kinder oder der Eltern aus ärztlichen, wissenschaftlichen oder seuchenhygienischen Gründen für erforderlich hält und der Verstorbene vor seinem Tode eine Sektion nicht ausdrücklich abgelehnt hat. Sie unterbleibt, wenn von den nächsten Angehörigen innerhalb von 15 Stunden nach Ableben Einspruch erhoben wird.« zit. nach Haas, L., Die Zulässigkeit klinischer Sektionen, NJW 1988, S. 2929 (2933).

85 Dazu: Georgii/Meliss, Häufigkeit klinischer Obduktionen unter der Widerspruchs- gegenüber der Zustimmungslösung an der Medizinischen Hochschule Hannover, Pathologe 13 (1992) S. $190 \mathrm{ff}$.

86 Drs. 6/585 vom 15.9.1972, die Regelungen zur Sektion sind abgedruckt in Linck, Gesetzliche Regelung von Sektionen und Transplantationen, JZ 1973, S. 759 (761). 
tete die damalige CDU-Fraktion des Berliner Abgeordnetenhauses mit einem Alternativvorschlag, der ebenfalls Regelungen der beiden Arten der Sektion beinhaltete. ${ }^{87}$

a) Entwurf des Berliner Senats von 1972 zur klinischen und anatomischen Sektion

Nach dem Senatsentwurfs sollte außer in den sonst durch Gesetz zugelassenen Fällen eine klinische Sektion vorgenommen werden können, wenn dies im Einzelfall im ärztlichen Interesse liegt, zur Klärung des Grundleidens, der Todesursache, zur Überprüfung einer Diagnose oder einer therapeutischen Maßnahme. Voraussetzung sollte sein, daß nach stattgehabter äußerer Leichenschau keine Anhaltspunkte für einen »nichtnatürlichen « Tod vorgelegen haben. Ferner sollte der Verstorbene in die Leichenöffnung eingewilligt haben oder - wenn er ihr nicht widersprochen hat - den Angehörigen eine Widerspruchsfrist von 24 Stunden eingeräumt werden (erweiterte Widerspruchslösung). Der Kreis der Angehörigen sollte durch Auflistung eingegrenzt werden. Die Entscheidung über das Vorliegen der oben genannten Voraussetzungen sollte bei den behandelnden Ärzten liegen, eine schriftliche Begründung sollte notwendig sein und eine Niederschrift über das Ergebnis der Leichenöffnung sollte auch Angaben zur Einhaltung der Widerspruchsfrist enthalten.

b) Alternativentwurf der CDU-Fraktion des Berliner Abgeordnetenhauses von 1973 zur klinischen und anatomischen Sektion

Der differenziertere Alternativentwurf der damaligen CDU-Fraktion wollte in gleicher Weise bei ärztlichem Interesse eine klinische Sektion zulassen nach abgeschlossener äußerer Leichenschau, soweit bei dieser Leichenschau keine Anhaltspunkte für einen »nichtnatürlichen« Tod gefunden wurden. Eine Sektion entgegen dem Glauben und der Weltanschauung des Verstorbenen sollte ausdrücklich für unzulässig erklärt werden. Der Verstorbene oder einer seiner totensorgeberechtigten Hinterbliebenen sollte der Sektion ausdrücklich zugestimmt haben, die entsprechende Einwilligung sollte in schriftlicher Form oder in Gegenwart von mindestens zwei Zeugen erklärt worden sein (erweiterte Einwilligungslösung). Vorgesehen war ebenfalls, daß über die Sektion eine Niederschrift anzufertigen ist, aus der hervorgehen sollte, daß die verlangten Voraussetzungen für eine Sektion gegeben waren. In einem eigenen Paragraphen wird ausdrücklich verlangt, daß entnommene Leichenteile - soweit nicht für den Zweck der Sektion deren Entnahme unbedingt erforderlich ist - dem Leichnam wieder eingefügt werden müßten. Auch wurde gesondert festgelegt, daß für die Einwilligung in eine Sektion keine Gegenleistung verlangt werden dürfe.

\section{Vorschläge der Bund-Länder-Arbeitsgruppe von 1978}

Eine Bund-Länder-Arbeitsgruppe befaßte sich neben der Transplantationsproblematik auch mit der Frage einer gesetzlichen Regelung der klinischen Sektionen. Obduktionen von außerhalb der Kliniken und Krankenhäuser Verstorbenen - wie sie gerade

87 Drs. 6/948 vom 22.6.1973, ebensfalls auszugsweise abgedruckt in Linck, JZ 1973, S. 759 (761). 
auch durch eine Verwaltungssektion erfaßt würden - wurden nicht berücksichtigt. In ihrem 1978 vorgelegten Kommissionsbericht wurden als Anlage I und Anlage II Vorschläge zur Änderung des $§ 168$ StGB unterbreitet. Dabei wurde eine Mehrheitslösung (Entwurf I) und eine Minderheitslösung (Entwurf II) präsentiert. ${ }^{88}$

a) Mehrheitslösung der Bund-Länder-Arbeitsgruppe von 1978

Nach dem Entwurf der Mehrheitslösung (Entwurf I) sollte ein $\S 168 \mathrm{c}$ in das StGB eingefügt werden, nach dem die Sektion unter den näher geregelten Voraussetzungen ohne strafrechtliche Konsequenzen bleiben sollte. Eine klinische Sektion sollte danach durchgeführt werden können bei »medizinischer Notwendigkeit«, es sei denn, ein Widerspruch des Verstorbenen selbst liege vor. Für die Totensorgeberechtigten waren nach diesem Entwurf keine eigenen Entscheidungsbefugnisse vorgesehen. Zur Begründung wird nach Darlegung der Bedeutung von Obduktionen ausgeführt, die Vorteile einer hohen Obduktionsfrequenz »lassen nach der Mehrheitsmeinung eine derartige Pflichtsektionslösung geboten erscheinen. ${ }^{89}$ Damit favorisierte die Mehrheit der Bund-Länder-Gruppe eine enge Widerspruchslösung.

b) Minderheitslösung der Bund-Länder-Arbeitsgruppe von 1978

Auch die Minderheitslösung (Entwurf II) sah die Einführung eines § 168c in das Strafgesetzbuch vor. Danach sollte primär die Einwilligung des Verstorbenen oder seiner Angehörigen in die Obduktion erforderlich sein. Bei Fehlen eines entgegenstehenden Willens des Verstorbenen sollte aber eine klinische Sektion auch dann möglich sein, wenn keiner der nächsten Angehörigen Widerspruch erhob (erweiterte Widerspruchslösung). ${ }^{90}$

\section{Gesetzliche Regelung der klinischen Sektion in der Freien Hansestadt Bremen}

In $\S 11$ des »Gesetzes über das Leichenwesen « ${ }^{91}$ hat die Freie Hansestadt Bremen eine Vorschrift zur klinischen Sektion eingeführt. Danach kann eine klinische Sektion durchgeführt werden, wenn der Leichenschauarzt dies schriftlich beantragt und der Verstorbene zu Lebzeiten eine Einverständniserklärung abgegeben hat. Als Einverständniserklärung gilt auch eine bei der Krankenhausaufnahme ausdrücklich abgegebene Erklärung. Damit wird eine dem AGBG unterfallende sogenannte Sektionsklausel im Krankenhausaufnahmevertrag ausdrücklich als nicht ausreichend angesehen. Ohne Einverständniserklärung und bei fehlendem Widerspruch des Verstorbenen kann die Obduktion vorgenommen werden, wenn die Hinterbliebenen nach vorheriger Information über die geplante Obduktion und über die Möglichkeit eines

88 Bericht der Bund-Länder-Arbeitsgruppe zur Vorbereitung einer gesetzlichen Regelung der Transplantation und Sektion. BMJ - Bibliothek - Q 80a48K - ohne Angabe von Erscheinungsort und Erscheinungsjahr, S. 24 und S. 25.

89 Bericht der Bund-Länder-Arbeitsgruppe, S. 24.

90 Bericht der Bund-Länder-Arbeitsgruppe, Anlage 2, Entwurf II, S. 37.

91 Gesetz über das Leichenwesen vom 27. Oktober 1992, GBl. der Freien Hansestadt Bremen 1992, S. 627-633. 
Widerspruchs innerhalb von 24 Stunden von ihrer Widerspruchsmöglichkeit keinen Gebrauch machen (erweiterte Widerspruchslösung). Der Widerspruch erfolgt ohne Angabe von Gründen. Die Reihenfolge der zu befragenden Angehörigen ist gesondert geregelt. Danach ist zuerst der Ehegatte beziehungsweise die Ehegattin zu befragen, anschließend die volljährigen Kinder oder volljährige Geschwister. Die klinische Obduktion ist nur dann zulässig, wenn dem Antrag des Leichenschauarztes eine schriftliche Erklärung beigefügt ist, daß die Hinterbliebenen sowohl informiert als auch auf die Möglichkeit des Widerspruchs hingewiesen worden sind.

\section{Vorschlag der Deutschen Gesellschaft für Medizinrecht (DGMR e.V.)}

Bezogen auf die Todesfälle in Kliniken und Krankenhäusern, also bei anstehender klinischer Sektion, hat sich die Deutsche Gesellschaft für Medizinrecht (DGMR e.V.) für eine gesetzliche Verankerung der Informationslösung ausgesprochen. ${ }^{92}$ Die Information der Angehörigen über die geplante Obduktion wird danach verbunden mit der Aufforderung, innerhalb einer gesetzten Frist der Sektion zu widersprechen. ${ }^{93}$ Wird nicht widersprochen, also bei stillschweigender Duldung, so soll die Einwilligung in die Obduktion als erteilt gelten. Bedenken ergeben sich auch hier wegen eines Verstoßes gegen den Grundsatz, daß einfaches Schweigen keine Willenserklärung ist, wer schweigt bringt eben weder Zustimmung noch Ablehnung zum Ausdruck. ${ }^{94}$ Zudem wäre anläßlich der Information über die anstehende Obduktion zugleich über das Recht zum Widerspruch aufzuklären. De facto ergäbe sich mit dieser Informationslösung eine erweiterte Widerspruchslösung.

\section{Regelung in der »Anordnung über die ärztliche Leichenschau« der früheren DDR}

Für Leichenöffnungen galt in der früheren DDR und bis zur teilweise erst kürzlich erfolgten Ablösung durch Landesrecht $\S 8$ der »Anordnung über die ärztliche Leichenschau « vom 4. Dezember 1978. ${ }^{95}$ Danach mußte eine Leichenöffnung vorgenommen werden bei Verstorbenen ohne feststellbare Todesursache, bei verstorbenen Schwangeren und Wöchnerinnen, bei Totgeborenen, bei verstorbenen Kindern und Jugendlichen bis zum 16. Lebensjahr, bei unbekannten Toten und auf Wunsch der Angehörigen. Neben dieser »Muß«-Regelung gab es eine »Soll«-Regelung. Nach dieser sollte eine Leichenöffnung vorgenommen werden bei Verstorbenen mit »nichtnatürlichem« Tod und »ungeklärter« Todesart, bei Verstorbenen mit einer meldepflichten übertragbaren Krankheit, einer Berufskrankheit oder einer melde-

92 Empfehlung der DGMR, MedR. 1991, S. 76 bezogen auf die klinische Sektion, jedoch ausdrücklich verbunden mit der Aufforderung an den Gesetzgeber, er möge eine Regelung treffen, bei der auch Todesfälle außerhalb der Klinik berücksichtigt werden, um »ärztlichfürsorgerischen und epidemiologischen« Anliegen Rechnung zu tragen.

93 Einbecker Empfehlungen zu Rechtsfragen der Obduktion, MedR. 1991, S. 76, Ziff. IV.

94 Palandt-Heinrichs, BGB, Einf. v. § 116, Rdnr.7; allerdings ist fraglich, ob die bloße (stillschweigende) Gestattung einer Obduktion gleichzusetzen ist mit der Interpretation des Schweigens als rechtsgeschäftlicher Willenserklärung.

95 GBl. DDR 1979, S.4. 
pflichtigen Geschwulstkrankheit sowie bei Verdacht auf das Vorliegen einer dieser Krankheiten. Ferner bei Verstorbenen, bei denen innerhalb der letzten 4 Wochen vor Eintritt des Todes eine Impfung vorgenommen wurde, bei Verstorbenen mit transplantierten oder implantierten Organen, bei begründetem wissenschaftlichen Interesse und bei Verstorbenen mit implantiertem Herzschrittmacher. Die Obduktion mußte vom Leichenschauarzt beim zuständigen Kreisarzt beantragt werden, dieser hatte bei Vorliegen der Voraussetzungen die Sektion anzuordnen. Für die zuerst genannten Fälle bestand damit eine Pflichtsektion, im übrigen eine Soll-Vorschrift. Religiöse oder weltanschauliche Vorbehalte gegen eine Obduktion wurden nicht berücksichtigt, der Wille des Verstorbenen wurde - jedenfalls nach dem Wortlaut der Anordnung - nicht beachtet, ebenso wurde das Totensorgerecht der Hinterbliebenen ignoriert.

\section{6. »Indikationslösung« des 93. Deutschen Ärztetages von 1990}

Eine Regelung ähnlich der in der ehemaligen DDR wurde vom 93. Deutschen Ärztetag im Mai 1990 gefordert. Der kurze Beschluß lautet: »Der Vorstand der Bundesärztekammer wird aufgefordert, den Bundesgesetzgeber zu bitten, ein Obduktionsgesetz $z u$ beraten und zu beschließen. Grundlage eines Obduktionsgesetzes soll eine indikative Lösung sein, die bewährten Regelungen, wie z.B. in der DDR und Österreich folgt. ${ }^{96}$ Danach wäre in bestimmten Fällen zwingend zu obduzieren, zum Beispiel bei Tod einer Schwangeren, bei Tod im Anschluß an eine Operation etc.

\section{Gesetzliche Regelung des Obduktionswesens in Österreich}

Das östereichische Krankenanstaltengesetz ${ }^{97}$, auf das der 93. Deutsche Ärztetag 1990 hingewiesen hat, sieht in $\S 25$ Abs.1 vor, daß Verstorbene zu obduzieren sind, wenn die Obduktion »sanitätspolizeilich oder gerichtlich « angeordnet worden ist, wenn dies zur Wahrung öffentlicher oder wissenschaftlicher Interessen angezeigt ist, insbesondere bei diagnostischen Unklarheiten oder nach einem vorgenommenen operativen Eingriff. ${ }^{98}$ Diese in Österreich seit langem geltende und akzeptierte Regelung bedeutet ihrem Wortlaut nach die Etablierung einer Pflichtsektion (»sind « zu obduzieren). Eine normative Berücksichtigung religiös-weltanschaulicher Vorbehalte gegen eine Sektion findet nach dem Wortlaut der Vorschriften nicht statt, ebensowenig sieht die Vorschrift selbst eine Einbeziehung der Hinterbliebenen vor.

96 93. Deutscher Ärztetag, Entschließungen zum Tagesordnungspunkt V - Obduktionsgesetz. DÄ (87) 1990, S. 1121 (1136).

97 Krankenanstaltengesetz vom 15.7.1920, Staatsgesetzblatt für die Republik Österreich 1920, Teil 2, 1376 ff., der die Frage der Obduktionen regelnde $\S 31$ dieses Gesetzes wurde unverändert übernommen in $\S 25$ des Gesetzes vom 7.1.1957, BGBl. für die Republik Österreich 1957, Stück 1.

98 Ebenso das Gesetz über das Leichen- und Bestattungswesen im Land Vorarlberg, Vorarlberger Landesgesetzblatt, Jahrgang 1969, 22. Stück, Nr.58 in § 12 Abs.3. 
8. Neuere landesrechtliche Regelungen (z.B. in Berlin, Hamburg, Nordrhein-Westfalen, dem Saarland und Thüringen ${ }^{99}$ )

Die neueren landesgesetzlichen Regelungen verlangen für die klinische Sektion teils eine erweiterte Zustimmungslösung (Nordrhein-Westfalen - $\$ 10$ Abs.2; Thüringen $\S 8$ Abs.3), teils eine erweiterte Widerspruchs- bzw. Informationslösung (Hamburg $-\S$ 4 Abs.3 Nr.2; Berlin- $§ 3$ Abs.2 Nr. 3; Saarland - § 45 Abs.3 Nr.3; Bremen - $§ 11$ Abs.1). Als weitere Besonderheiten sind einerseits die Bremer Regelung einer »außergerichtlichen Obduktion« gem. § 12 brem. Gesetz über das Leichenwesen zu nennen. Danach kann die Staatsanwaltschaft durch die zuständige Behörde eine außergerichtliche Obduktion durchführen lassen. Darüberhinaus bestimmt $§ 20$ a des brem. Gesetzes, dass eine Feuerbestattung vorgenommen werden darf, wenn die Todesursache nach der Leichenschau und ggf. nach Rücksprache mit dem behandelnden Arzt »mit der notwendigen Sicherheit« festgestellt wurde. Gelingt eine solche Feststellung nicht, dann »ist « vom Gerichts- oder Amtsarzt eine Obduktion »anzuordnen «! Andererseits findet sich im Hamb. Sektionsgesetz sowohl für die klinische Sektion als auch für die rechtsmedizinische Sektion die Möglichkeit der Durchführung einer Obduktion auch ohne vorliegende ausdrückliche Einwilligung ( $\S 4$ Abs.2, 9 Abs.2), wenn

1. die Patientin oder der Patient eine schriftliche Erklärung zur Sektion krankheitsbedingt nicht geben konnte und

2. eine Einwilligung anderer Personen nicht vorliegt, insbesondere Angehörige oder eine bevollmächtigte Person binnen 24 h nach dem Tode der Patientin oder des Patienten nicht erreicht und befragt werden konnten, und

3. die Sektion aus ärztlicher Sicht als so dringend zur Fürsorge für die Hinterbliebenen oder zur Qualitätssicherung anzusehen ist, dass bei Abwägung das Interesse an ihrer Durchführung die fehlende Einwilligung der Betroffenen überwiegt.

Anordnungsbefugt sind jeweils die Leiterin und der Leiter der Pathologie im Einvernehmen mit der Ärztlichen Direktorin oder dem Ärztlichen Direktor bzw. die Leiterin oder der Leiter des Instituts für Rechtsmedizin.

\section{Fazit}

Bei zusammenfassender Betrachtung der dargelegten Entwicklung und aktuellen Situation im Leichenschau- und Obduktionsrecht ergibt sich ein wenig erfreuliches Bild:

1. Die häufig beklagte fehlende Vereinheitlichung des Leichenschau- und Obuktionsrechts in der Bundesrepublik Deutschland besteht fort.

99 Vgl. Gesetz Nr. 1535 über das Friedhofs-, Bestattungs- und Leichenwesen, Amtsbl. d. Saarlandes v. 11. Dez. 2003, S. 2920 ff.; Thüringer Bestattungsgesetz v. 19. Mai 2004, GVB1. S. 505 f.; Gesetz über das Friedhofs- und Bestattungswesen (BestG NRW) v. 17. Juni 2003, GVOBL. Nr. 29 v. 30. Juni 2003, S. 313 ff.; Gesetz zur Regelung des Sektionswesens und therapeutischer Gewebeentnahmen (Sektionsgesetz) v. 7. März 1997, GVOBl. Berlin, 52. Jahrgang, Nr.32, 237 ff.; Gesetz zur Regelung von klinischen, rechtsmedizinischen und anatomischen Sektionen (Sektionsgesetz) v. 9. Februar 2000, Hamburgisches GVOBl. 5, 38 ff.; Gesetz über das Leichenwesen v. 27. Oktober 1992, brem. GBl. Nr. 52, 627 ff. 
2. Eine Vereinheitlichung des Leichenschaurechts (einschließlich der Formulare für die Todesbescheinigungen) mit einer einheitlichen Klassifikation der Todesart (natürlich, nichtnatürlich, ungeklärt) fehlt weiterhin.

3. Einen neutralen Leichenschauarzt wird es auch zukünftig nicht geben, so dass etwa nach einem letalen Behandlungsfehler der den Tod verursachende Arzt auch die Todesbescheinigung ausstellen kann und verständlicherweise geneigt sein wird, einen natürlichen Tod anzugeben.

4. Für die klinische Sektion gibt es nach Landesrecht teils eine erweiterte Zustimmungslösung, teils eine erweiterte Widerspruchs- bzw. Informationslösung. Soweit landesgesetzliche Regelungen fehlen, bleiben als (unsichere) Rechtsgrundlage die nach dem ABGB zu beurteilenden Krankenhausaufnahmeverträge. Zur Hebung der Sektionsquote ist zumindest eine erweiterte Widerspruchs- bzw. Informationslösung wünschenswert. ${ }^{100}$

5. Für Todesfälle außerhalb von Kliniken und Krankenhäusern wird die Möglichkeit einer behördlich unter definierten Voraussetzungen anordbaren Verwaltungssektion (z.B. auf dem Boden einer erweiterten Widerspruchslösung) praktisch nicht von den Landesgesetzgebern aufgegriffen (Ausnahmen: Bremen und Hamburg). Verfassungsrechtliche Bedenken gegen eine derartige Verwaltungssektion zur Klärung allein medizinisch unklarer Todesfälle überzeugen nicht. ${ }^{101}$

6. Differenziertere Regelungen für therapeutische Gewebeentnahmen anlässlich einer Obduktion finden sich - außer in Berlin - nicht.

7. Die zu beklagenden Nachteile für die Todesursachenstatistik, bei der Aufdeckung nichtnatürlicher Todesfälle, für die ärztliche Fort- und Weiterbildung usw. bleiben bei einer im internationalen Vergleich extrem niedrigen Obduktionsquote bestehen.

8. Diese niedrige Obduktionsquote gefährdet mittlerweile eine ordnungsgemäße Aus- und Weiterbildung zum Facharzt für Pathologie; klinisch-pathologische Konferenzen auf der Grundlage von Obduktionsbefunden finden immer seltener statt, es fehlt eine Qualitätskontrolle zum Nutzen der (lebenden) Patientinnen und Patienten.

Nicht weil es bequem ist, sondern um der Sache willen soll hier nicht auf die Forderung verzichtet werden, die Kompetenz für das Leichenschau- und Obduktionsrecht wie für das Transplantationsrecht auf den Bund zu verlagern. Dies in der Hoffnung, dass dann eine befriedigendere bundeseinheitliche Regelung in einem Bundesgesetz möglich sein wird.

100 Verfassungsrechtliche Bedenken dagegen dürften nicht überzeugen, hat doch das BVerfG die Möglichkeit des Widerspruchs gem. § 3 Abs.2 Nr.1 TPG gegen eine Organentnahme im Transplantationsrecht ausreichen lassen; eine andere Einschätzung ist daher für das Obduktionsrecht nicht zu erwarten; vgl. BVerfG, 1. Kammer des Ersten Senats, Beschl. v. 14.10.1998 - 1 BvR 1526/98 - NJW 1999, 858.

101 Dazu ausführlich Dettmeyer, Verfassungsrecht und Verwaltungssektion, Fn. 13. 